\title{
Biodentine: novel endodontic material for single step apexification: A Case report.
}

SADJ August 2018, Vol 73 no 7 p452 - p455

Pradeep K', P Mohata ${ }^{2}$, RV Butula ${ }^{3}$

\begin{abstract}
The management of an immature tooth with pulpal necrosis and periapical pathology poses a great endodontic challenge. Treatment options comprise the conventional apexification procedure with and without apical barriers. The use of an apical barrier in cases with open apices has gained popularity in recent years.

The conventional apexification using calcium hydroxide has certain drawbacks such as the need for long term therapy to enable barrier formation. The recent trend is to form a fragile dentinal bridge as an artificial apical stop. Even though the current gold standard material for the apical stop is Mineral trioxide aggregate (MTA), a large number of novel materials have been studied for this purpose. Biodentine is a new calcium silicate based material, recently introduced as a dentine substitute, applicable whenever original root dentine is damaged. Biodentine was chosen for apexification because of its superior physical properties including short setting time, solubility, and easy handling characteristics. This case report describes single visit apexification in a maxillary central incisor with a necrotic pulp and an open apex using Biodentine as an apical barrier. The successful clinical outcome in this case is encouraging for the use of Biodentine as an apical plug in single visit apexification procedures.
\end{abstract}

Key Words: apexification, apical barrier, Biodentine

\section{INTRODCTION}

The completion of root development and closure of the root apex occurs up to three years following eruption of the tooth. ${ }^{1}$ If, during root formation, the tooth is affected by caries, trauma or other pulpal pathoses, dentin formation is interrupted and root development will cease. ${ }^{1}$ Consequently, the root canal is wide, with thin and weak walls, and the apex remains open. A further

1. Pradeep K: Department of Conservative Dentistry \& Endodontics, Manipal College of Dental Sciences, Manipal, Karnataka, India.

2. Payaswvi Mohata: Post Graduate student, Department of Conservative Dentistry \& Endodontics, Manipal College of Dental Sciences, Manipal, Karnataka, India.

3. Roshni Veena Butala: Post Graduate student, Department of Conservative Dentistry \& Endodontics, Manipal College of Dental Sciences, Manipal, Karnataka, India.

Corresponding author

Pradeep K:

Associate professor, Department of Conservative Dentistry \&

Endodontics, Manipal College of Dental Sciences, Manipal, Karnataka, India. E-mail: endopradeep@gmail.com

\section{ACRONYM \\ MTA: Mineral trioxide aggregate}

complication is that the dentinal tubules are wide, allowing the penetration of bacteria and irritants. ${ }^{2}$ These features make root canal instrumentation difficult and prevents achievement of a proper apical stop. In order to permit condensation of root canal filling material and promote apical sealing, it is important to create an artificial barrier or to stimulate closure of apical foramen with calcified tissue. Apexification is defined as 'a method to induce a calcified barrier in a root with an open apex or the continued apical development of an incomplete root in a tooth with a necrotic pulp. ${ }^{3}$ Whilst several procedures utilizing different materials have been proposed to induce root end barrier formation, calcium hydroxide has gained the widest acceptance. ${ }^{4}$ Although calcium hydroxide has a predictable outcome in apical barrier formation, the quality of the bridge and the time taken for bridge formation is not so predictable. $5,6,8,9$ Considering the various drawbacks associated with calcium hydroxide apexification, the use of the apical plug method seems to be a suitable alternate treatment plan for these cases.

Among the alternative materials mentioned, Mineral Trioxide Aggregate (MTA) is currently considered most promising because of its superior biocompatibility and lower cytotoxicity due to its alkaline $\mathrm{pH}$. Moreover, the presence of calcium and phosphate ions result in a capacity to attract blastic cells and hence promote favourable conditions for cementum deposition. ${ }^{10}$ However MTA has certain disadvantages including high solubility, prolonged setting time (approximately 2 hours and 45 minutes) and difficult handling characteristics. ${ }^{2}$

These disadvantages have necessitated the search for more ideal materials, having adequate biological and mechanical properties. Recently, a new calcium-silicate based material, Biodentine (Septodont, Saint-Maur-desFossés, France), has been formulated with the intention of preserving the properties and clinical applications of MTA without its negative characteristics. Biodentine is superior to MTA as its consistency is better suited to clinical use, ensuring better handling and safety, the material does not require a two-step obturation and, as the setting is faster; there is a lower risk of bacterial contamination. ${ }^{2}$

The aim of the present case report is to report the use of Biodentine in the successful closure of the root apex in a 
pulpless permanent maxillary central incisor with a wide open apex.

\section{CASE REPORT}

A 28-year old male patient reported to the Department of Conservative Dentistry and Endodontics with the chief complaint of a fractured and discoloured upper left central incisor (Figure 1a). He confirmed a history of trauma 10 years previously. The patient did not recall any history of swelling or pus discharge. The medical and dental histories were uncomplicated. Intraoral examination revealed generalized dental fluorosis with an Elli's Class IV fracture and discolouration on tooth no 21. Palpation and percussion test of the involved tooth did not reveal any tenderness. The tooth was not mobile and periodontal probing around the tooth was within physiological limits. Electric pulp testing (Parkell Electronics Division, Farmingdale, NY, USA) and thermal tests (Heat and Cold tests) of the involved tooth gave no response, whereas responses were obtained on the adjacent normal teeth. Detailed radiographic examination of the tooth revealed a wide canal with an open apex and a marked radiolucency periapically (Figure 1b). Evidently, development of the tooth had been interrupted by the trauma suffered years ago. A small healing sinus tract was seen near the position of the apex. Based on the history and the radiographic findings, a provisional diagnosis of chronic periapical abscess was made. The available treatment options were discussed with, and informed consent was obtained from, the patient. Root canal therapy with calcium hydroxide dressing, followed by apexification with

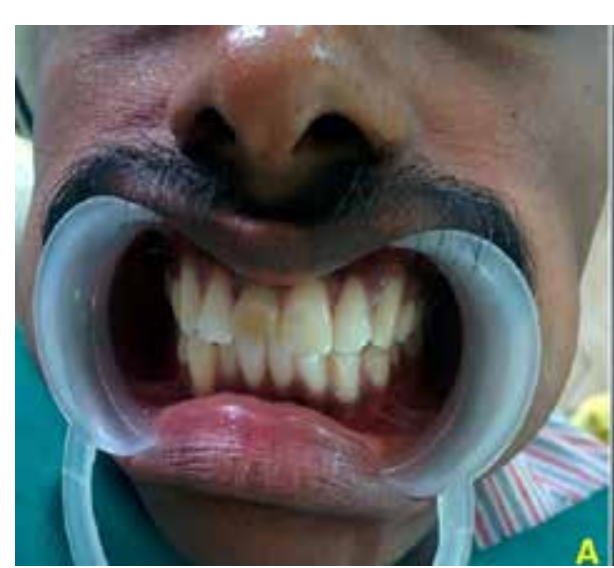

Figure 1a

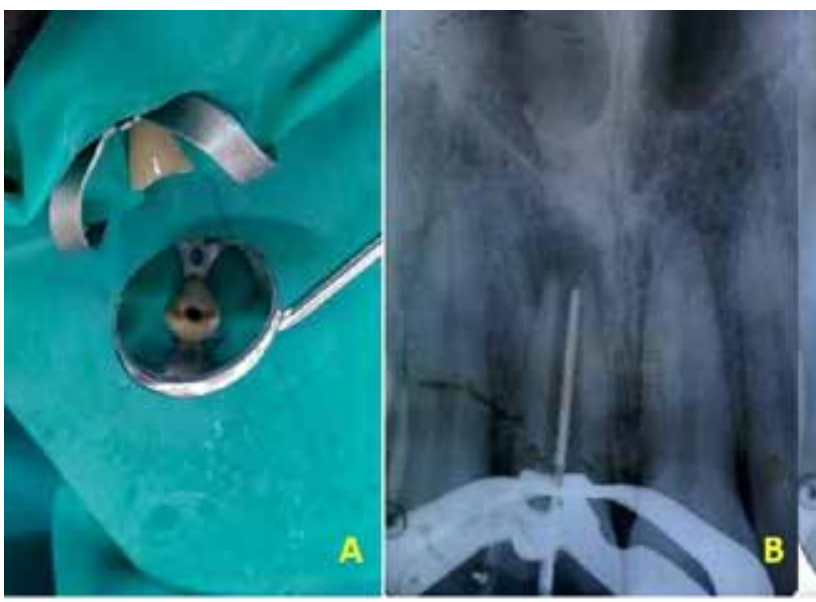

Figure 2a

Figure 2b

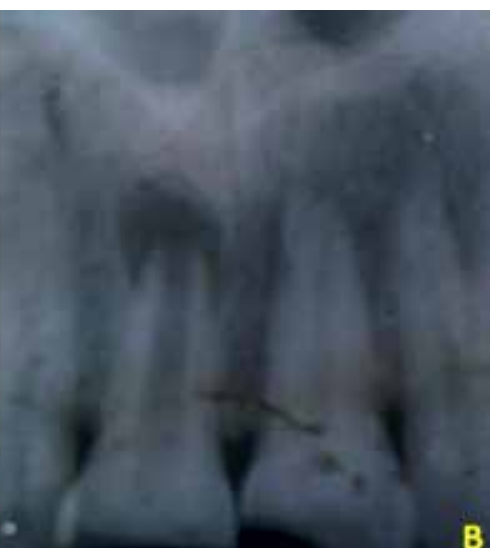

Figure 1b
Biodentine was selected. After rubber dam application under local anaesthesia, the pulp cavity was opened to allow access (Figure 2a). Endodontic working length was established (Figure 2b). Biomechanical preparation was completed using No 80 stainless steel K- file (MANI, INC., Utsunomiya Tochigi, Japan) under copious irrigation with $5.25 \% \mathrm{NaOCl}$ (Cmident, India). Irrigation was carried out passively with side-vented irrigation needles ( R C Twents irrigation needle, Prime Dental Products Pvt. Ltd, Mulund Mumbai), keeping the points $1 \mathrm{~mm}$ short of the radiographic apex. Calcium hydroxide intracanal medicament (RC Cal, Prime Dental) was placed. The patient was recalled after three weeks and the involved tooth was found to be asymptomatic. The access cavity was reopened, the canal copiously irrigated with 5.25\% $\mathrm{NaOCl}$ solution and then dried with sterile paper points. Biodentine was mixed according to the manufacturer's protocol and pellets were placed with a plugger until a thickness of $5 \mathrm{~mm}$ had been achieved (Figure 2c). A sterile cotton ball was placed in the canal for 15 minutes and then the root canal was obturated by the thermo plasticized Gutta-percha technique (Obtura - Spartan) (Figure 2d). Non vital bleaching was carried out on two subsequent appointments but no colour change was observed. The access cavity was then sealed with a composite restoration and the discoloured tooth was restored by an all-ceramic crown (Figure 3a). Follow up examinations were carried out at one month, three months, six months and one year after apexification (Figure 3b). During follow up periods the involved tooth was asymptomatic and the post-operative radiographs taken at one year showed remarkable healing of the osseous lesion.

\section{DISCUSSION}

It is not only the wide-open root apex presented by immature permanent teeth that poses unique challenges during endodontic procedures, but also the fragile weak dentin walls. ${ }^{10}$ The conventional apexifcation technique using calcium hydroxide requires at least three to four months and involves multiple appointments. Patient compliance with this protracted treatment protocol may be poor and many fail to return for scheduled appointments. The aim of

\section{Figure 2a}

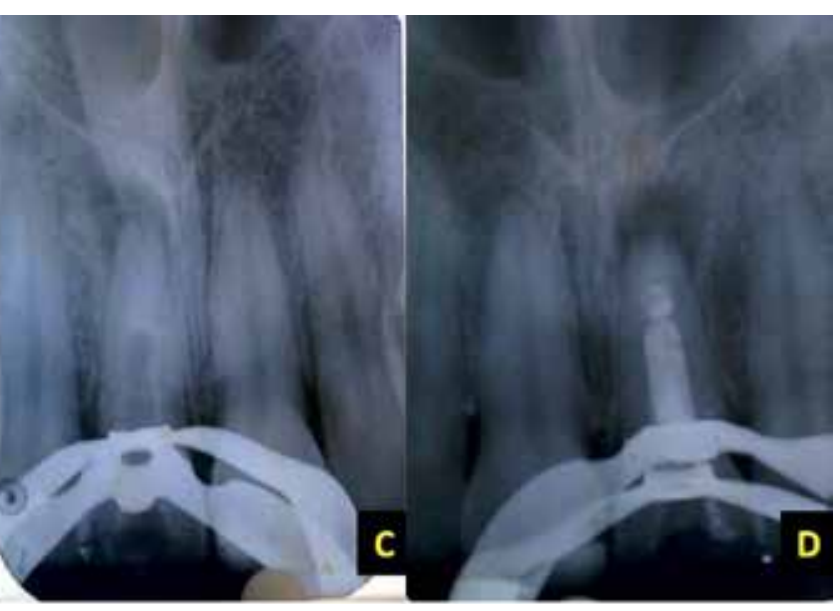

Figure 2c

Figure 2d 


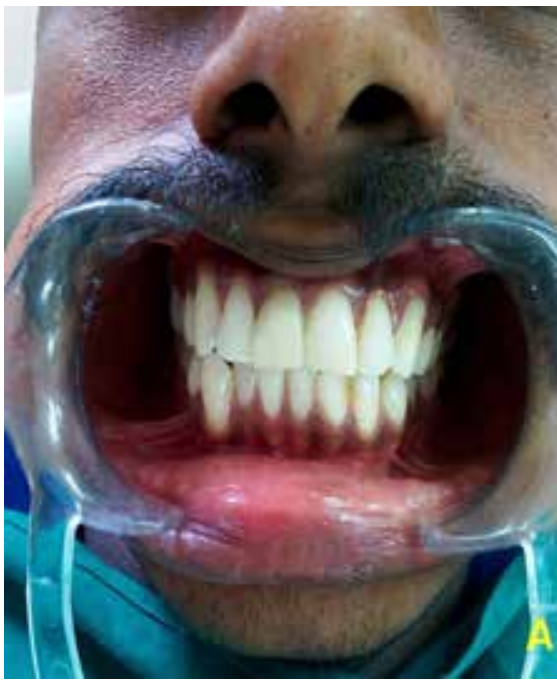

Figure 3a

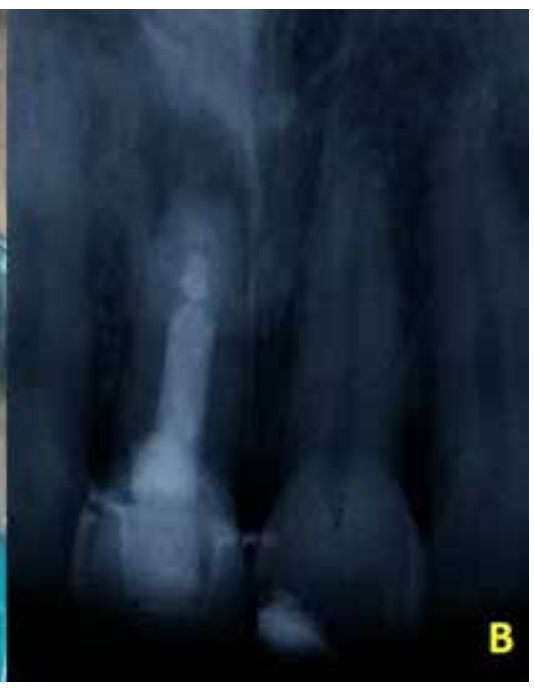

Figure $3 b$

Table 1: Composition and functions of constituents

\begin{tabular}{|lll}
\hline \multicolumn{2}{c}{ Constituents } & Function of constituents \\
\hline Powder & $\begin{array}{l}\text { Tri-calcium Silicate }\left(3 \mathrm{CaOSiO}_{2}\right. \\
\text { Di-calcium Silicate }\left(2 \mathrm{CaOSiO}_{2}\right)\end{array}$ & Main core material \\
\hline $\begin{array}{l}\text { Calcium Carbonate and Oxide } \\
\left(\mathrm{CaCO} \text { and } \mathrm{CaO}_{2}\right)\end{array}$ & Filler \\
\hline Iron Oxide $\left(\mathrm{FeO}_{2}\right)$ & Shade \\
\hline Zirconium Oxide $\left(\mathrm{ZrO}_{2}\right)$ & Radiopacifier \\
\hline Calcium chloride $\left(\mathrm{CaCl}_{2} \cdot 2 \mathrm{H}_{2} \mathrm{O}\right)$ & Accelerator \\
\hline Hydrosoluble polymer & Water reducing agent
\end{tabular}

the treatment described in this case report is to create in a single appointment an apical barrier which will prevent the penetration of toxins and bacteria into periapical tissues from the root canal. Technically, this barrier is also necessary to allow the compaction of root filling material. ${ }^{11,12}$ Whilst a higher success rate of apical barrier formation has been reported with the use of calcium hydroxide, long term follow up is essential. ${ }^{13}$ Previous studies have described the disadvantages of calcium hydroxide apexification which include failure to control infection, recurrence of infection and cervical fracture. ${ }^{12}$

Apexification using MTA provides an alternative treatment option in immature pulpless teeth. The long setting time of ProRoot MTA is a major problem of the material, apart from poor handling characteristics, discoloration potential (Gray MTA), low washout resistance and high material cost. ${ }^{14}$

Biodentine is a newly introduced (2011) bioactive dentine substitute based on "Active Biosilicate Technology." It is biocompatible, has mechanical properties similar to dentin, and has good sealing ability on dentinal surfaces. ${ }^{15}$ The detailed composition and the role of each ingredient are summarized in Table $1 .^{16}$

This case presentation has shown that Biodentine can be used as an effective alternative to MTA, even in the older patient. A study comparing microleakage of glass ionomer cement, MTA, and Biodentine found that the latter exhibits the least microleakage when used as a

retro-filling material. ${ }^{16}$ In addition, the material forms a chemicomechanical bonding with both tooth and composite, which reinforces the thin fragile immature roots. ${ }^{17}$ Its short setting time (9-12 $\mathrm{min})$ is attributed to the smaller particle size, the addition of calcium chloride as accelerator, and reduction in the amount of liquid required for setting. Calcium chloride improves its consistency, making its condensation in the canal more controlled, avoiding need for a matrix, and decreases the chances of the material going beyond the apex, making it safer and easier to handle than MTA. ${ }^{18}$ Its insolubility in saliva and the ability to withstand pressure of $400 \mathrm{gm} \mathrm{mm}-2$ within six minutes of setting makes it ideal for single visit apexification, negating the need for a second appointment for obturation. ${ }^{19}$

About et al. investigated the bio activity of biodentine on dentin. They concluded that biodentine induces dentin regeneration by stimulating pulp progenitor cells. ${ }^{20}$ Studies by Han and Okiji concluded that calcium and silicon uptake by root dentin and the thickness of the hard tissue barrier formed in the case of biodentine are comparable to pro-root MTA. ${ }^{21}$ Due to its superior material properties, Biodentine has a distinct advantage over its closest alternatives in the treatment of teeth with open apex. The material is still under study and several advancements in its clinical applications may be expected in the near future.

\section{CONCLUDING REMARKS}

Single visit apexification with biocompatible materials such as Biodentine can be considered an effective treatment option for teeth presenting with open apices. Many of the drawbacks of relying on calcium hydroxide and MTA have been overcome by the use of Biodentine, which is accompanied by superior results. The material has great potential in the management of a tooth with an open apex, particularly in its capacity to achieve biomimetic mineralisation. However further research is required to explore the scope of the clinical applications of this material.

\section{References}

1. Bhasker SN. Orban's Oral Histology and Embryology. 11th ed. St. Louis: Mosby-Year Book; 1991. p382.

2. Balasubrahmaniam A, Sabna BS, Ramesh Kumar M, et al. One visit apexification using a novel dental material, Biodentine - A case report. IOSR Jnl Dent Med Sci. 2014; 13(5): 90-2.

3. American Association of Endodontists: Glossary of Endodontic Terms (Visited in 2015. Accessible at URL: http://www.aae. org/ (updated 2013 Nov 6).

4. Nagaveni NB, Umashankara KV, Radhika NB, Manjunath S. Successful closure of the root apex in non-vital permanent incisors with wide open apices using single calcium hydroxide $(\mathrm{CaOH})$ dressing report of two cases. J Clin Exp Dent. 2010; 2:26-9.

5. Sheehy EC, Roberts GJ. Use of calcium hydroxide for apical barrier formation and healing in nonvital immature permanent teeth: a review. Br Dent J. 1997; 183: 241-6. 
6. Felippe MC, Felippe WT, Marques MM et al. The effect of renewal of calcium hydroxide paste on the apexification and periapical healing of teeth with incomplete root formation. Int Endod J. 2005; 38: 436- 42.

7. Metzger Z, Solomonov M, et al. Calcium hydroxide retention in wide root canals with flaring apices. Dent Traumatol. 2001; 17: 86-92.

8. Andreasen JO, Farik B, et al. Long-term calcium hydroxide as a root canal dressing may increase risk of root fracture. Dent Traumatol. 2002; 18: 134-7.

9. Binnie $\mathrm{WH}$, Rowe $\mathrm{AH}$. A histological study of periapical tissues of incompletely formed pulpless teeth filled with calcium hydroxide. J Dent Res. 1973; 52: 1110-6.

10. Apexification: a review. Dent Traumatol. 2005; 21: 1-8.

11. Komabayashi T, Spångberg LS. Comparative analysis of the particle size and shape of commercially available mineral trioxide aggregates and Portland cement: A study with a flow particle image analyzer. J Endod. 2008; 34: 94-8.

12. Trope M. Treatment of immature teeth with non-vital pulps and apical periodontitis. Endotopic. 2007; 14: 51-9.

13. Khetarpal A, Chaudhary S, Talwar S, Verma M. Endodontic management of open apex using Biodentine as a novel apical matrix. Indian J. Dent. Res. 2014; 25: 513-6.

14. Maroto M, Barbería E, Planells P, et al. Treatment of a non-vital immature incisor with mineral trioxide aggregate (MTA). Dent Traumatol. 2003; 19: 165-9.

15. Parirokh $M$, Torabinejad M. Mineral trioxide aggregate: $A$ comprehensive literature review - Part III: Clinical applications, drawbacks, and mechanism of action. J Endod. 2010; 36: 400-13.

16. Leiendecker AP, Qi YP, Sawyer AN, et al. Effects of calcium silicate-based materials on collagen matrix integrity of mineralized dentin. J Endod 2012; 38: 829-33.

17. Malhotra S, Hegde MN. Analysis of marginal seal of ProRoot MTA, MTA Angelus Biodentine, and glass ionomer cement as root-end filling materials: An in vitro study. Jnl Oral Res Rev.2015;44-9.

18. Septodont Biodentine Active Biosilicate Technology Scientific File. 2010. (Visited on 2015). Acessed URL from http//www. septodont.fr/fichiers_upload/biodentinescientificfile.pdf.

19. Kokate SR, Pawar AM. An in vitro comparative stereomicroscopic evaluation of marginal seal between MTA, glass ionomer cement and Biodentine as root end filling materials using $1 \%$ methylene blue as tracer. Endodontol. 2012; 2: 36-42.

20. About I, Laurent P, Tecles O. Bioactivity of Biodentine, a Ca3SiO5-based dentine substitute. Oral session. IADR Congress July 2010, Barcelona, Spain.

21. Han L, Okiji T. Uptake of calcium and silicon released from calcium silicate-based endodontic materials into root canal dentine. Int Endod J 2011; 44: 1081-7. 